\title{
Amorphous Computing
}

\author{
Matthew N. O. Sadiku, Yu Zhou, and Sarhan M. Musa \\ Roy G. Perry College of Engineering \\ Prairie View A\&M University \\ Prairie View, TX 77446
}

\begin{abstract}
The Amorphous computer consists of a large number of tiny interacting computing elements, each with a CPU, memory, and means of communication. An amorphous computing medium is a system of irregularly placed, asynchronous, locally interacting computing elements or agents. Each element is programmed identically and can communicate with a few neighbors. This paper provides a brief introduction on Amorphous computing.
\end{abstract}

Key words: Amorphous Computing, Cellular Computing.

\section{INTRODUCTION}

Ideas from computing and biology have been combined to create amorphous computing. The term "amorphous computing"(AC) was coined by a research team at MIT in 1996 They regarded AC as a bio-inspired novel computing paradigm that mimics the way organisms compute [1].

Amorphous computing refers to computational systems that use very large numbers of identical, parallel processors each having limited computational ability. The study of amorphous computing aims to identify useful programming methodologies that will enable us to engineer the emergent behavior of a myriad, locally interacting computing elements. The elements or agents run asynchronously, are interconnected in unknown manner, communicate only locally, are identically programmed, and are lacking a rigidly defined architecture for sharing information. Each element has modest computing power and memory. Since the number of particles is assumed to be very large, the entire amorphous medium may be considered as a massively parallel computing system.

Amorphous computations can be found in many fields such as developmental biology, molecular biology, neural networks, and chemical engineering [2]. Recently, amorphous computing has attracted a great interest both as an alternative model of computing and as an inspiration to understand developmental biology. Development in electronics and microfrabrication has made it possible to produce huge number of almost-identical information-processing units at almost no cost.

\section{CONCEPT OF AMORPHOUS COMPUTING}

Amorphous computing differs from the traditional computations in many ways. The architecture of amorphous computers is random, whereas there is no room for randomness in classical models such as RAMs [3]. They consist of a set of elements and self-powered processors that can communicate wirelessly over a limited distance. The creation of order in an asynchronous, randomly structured network of cells is the mystery of amorphous computing. The idea of AC is to distribute computations to a large number of locally interacting processing elements rather than implementing sequentially basic instructions.

An amorphous computer consists of several identical processing elements. Each element has limited computing resources [4]. The elements are physically similar to sand particles and they are referred to as amorphous particles. The particles communicate through broadcast messages, which can only be heard within a radius $r$ of its source. This is illustrated in Figure 1 [5]. There is no guarantee that a message will reach its destination due to collisions [6]. A particle may not initially know the distances or directions to any of its neighbors but knows their IDs.

Amorphous computers tend to have the following properties [2]:

- Implemented by redundant, potentially faulty, massively parallel devices.

- Devices having limited memory and computational abilities. 
- Devices being asynchronous.

- Devices having no a priori knowledge of their location.

- Devices communicating only locally.

- Exhibit emergent or self-organizational behavior.

- Fault-tolerant, especially to the occasional malformed device.

Any framework with these properties lends itself for amorphous computation.

An amorphous computing medium consists of independent computational particles, all identically programmed and inspired by nature with computer science techniques for controlling complexity. An amorphous computer system (ACS) consists of a $\mathrm{CPU} / \mathrm{memory}$ core surrounded by programmable hardware which can be used to enhance the function of the core. Programmable hardware enables the I/O to be tailored to the application during design and fine tuned during system integration and test [7]. An ACS can either be simulated or implemented in hardware. The idea of amorphous computing is to develop engineering techniques to control, organize, and exploit the behavior of a set of programmable computing particles [5].

\section{APPLICATIONS}

The set of potential applications for amorphous computing is vast. Amorphous computing can deal with a variety of problems such as biology, sociology, computational geometry, self-reconfiguring computers or robot, cellular computing, logic system, programmable materials, smart paint, and smart materials. For example, smart materials involve mixing computational particles with bulk materials such as paint, gel, or concrete. The smart paint could be used to coat objects and then compute their shape, to sense the environment. Biological applications require nodes on sizes compatible with molecules or cells, which are complex and expensive to make using conventional top-down fabrication techniques.

\section{BENEFITS AND CHALLENGES}

One of the interesting properties of amorphous computers is that they are robust with respect to the loss of individual processing units or elements. The ability to tailor a single board to diverse needs is advantageous where logistics and fault tolerance are concerned.

A completely functional amorphous computer has not yet been constructed. This is due to the fact silicon-based fabrication techniques (used for microelectronic processors) are not specifically built for use in an amorphous computer. To fabricate molecular-scale electronic structures may require cellular computing in conjunction with amorphous computing.

\section{CONCLUSION}

Amorphous computing is a non-classical computing paradigm that relies on massively parallel computation by a large number of small elements weakly interacting together.

This is based on the existence of large numbers of inexpensive nodes with limited computational ability, limited memory capacity, and limited communication range. It is an emerging domain in computer science. We are at early stage in the development of amorphous computing.

\section{REFERENCES}

[1] D. Chu, D. J. Barnes, and S. Perkins, “Amorphous computing in the presence of stochastic disturbances," BioSystems, vol. 25, 2014, pp. 32-42.

[2] "Amorphous computing," Wikipedia, the free encyclopedia

https://en.wikipedia.org/wiki/Amorphous_computing

[3] J. Wiedermann and L. Petru, "On the universal computing power of amorphous computing Ssystems," Theory of Computing System, vol. 45, 2009, pp. 995-1010.

[4] R. Nadpal and D. Coore, "An algorithm for group formation in an amorphous Computer," Proceedings of the 10 ${ }^{\text {th }}$ International Conference on Parallel and Distributed Systems, 1998.

[5] E. D'Hondt, “Exploring the amorphous computing paradigm," Master's Thesis.

Vrije Universiteit Brussel, August 2000.

[6] J. Katzenelso, "Notes on amorphous computing,"

https://www.researchgate.net/publication/2242486_Notes_on_Amorphous_Computing

[7] N. W. Anderson, "Amorphous computer system architecture: A preliminary look," 


\section{ABOUT THE AUTHORS}

Matthew N.O. Sadiku is a professor at Prairie View A\&M University, Texas. He is the author of several books and papers. He is an IEEE fellow. His research interests include computational electromagnetics and computer networks.

Yu Zhou is a doctoral student at Prairie View A\&M University, Texas. He worked for Institute of Automation Shandong Academy of Sciences. His research interests are in natural language processing, image processing, machine learning, deep learning and autonomous underwater vehicle design.

Sarhan M. Musa is a professor in the Department of Engineering Technology at Prairie View A\&M University, Texas. He has been the director of Prairie View Networking Academy, Texas, since 2004. He is an LTD Sprint and Boeing Welliver Fellow.

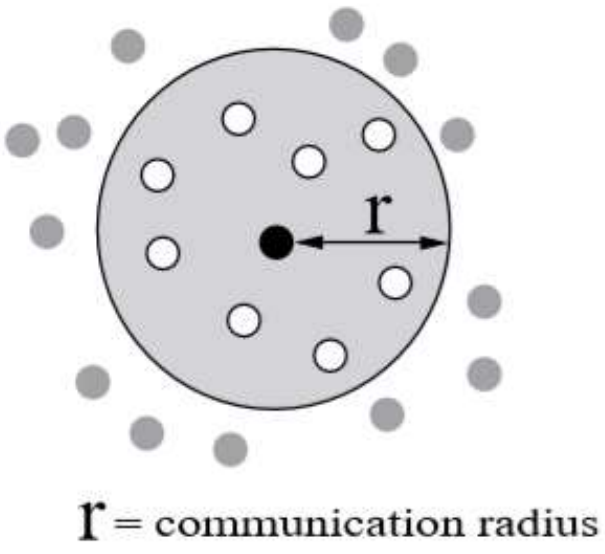

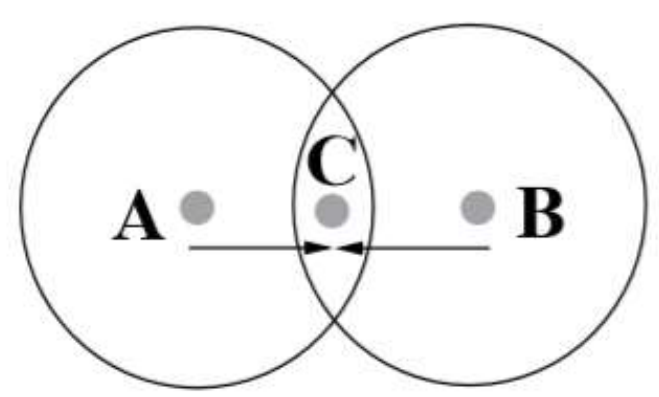

If $\mathrm{A}$ and $\mathrm{B}$ broadcast a message at the same time, $\mathrm{C}$ will detect a collision

Figure 1 The communication model [5]. 